\title{
Boundary-Layer Flow and Heat Transfer over a Rotating Porous Disk in a Non-Newtonian Williamson Nanofluid
}

\author{
N. Ambreen ${ }^{1, *}$, A. Rehman ${ }^{1}$, N. Sheikh ${ }^{1}$, S. Iqbal ${ }^{1}$, M. Zulfiqar ${ }^{2}$ \\ 'Department of Mathematics, University of Balochistan, Quetta, Pakistan; \\ nazish_ambreenmaths@yahoo.com,abdul_maths@yahoo.com, \\ naveed_maths@hotmail.com, saleem_iqbal61@yahoo.com \\ 2Department of Mathematics, Government College University, \\ Lahore, Pakistan; mzulfiqarshafi@hotmail.com
}

\begin{abstract}
Objectives: The present study analyses the effects of nanoparticles over steady, incompressible boundary-layer flow of non-Newtonian Williamson fluid flowing over a rotating porous disk. Methods/statistical analysis: The analysis used nonlinear partial differential equations associated with the non-Newtonian Williamson fluid flow and used an abridged, simplified version of ordinary differential equations under the Boussines q and boundary-layer approximations. The resulting system of nonlinear ordinary differential equations is then solved analytically using the homotopy analysis. Findings: The behavior of three non-dimensional velocity profiles and the non-dimensional temperature profile for important physical parameters like Williamson number, Prandtl number and surface suction/ injection parameters is tabulated and the results are graphed. Application/improvements: Nanoparticles like Copper, Alumina and titania are commonly used with liquid coolants to increase the heat-dissipating capability.
\end{abstract}

Keywords: Boundary-Layer Flow; Heat Transfer; Porous Disk; non-Newtonian; Williamson Nanofluid

\section{Introduction}

The study of fluid flow, particularly fluid flow for non-Newtonian fluids, gained immense popularity in industrial and engineering applications due to their extensive utilization. Common non-Newtonian fluid sare basically pseudoplastic fluids that have various industrial applications; they are used in production of emulsion sheets, polymer sheet extrusion, and are also used for aiding the flow of plasma and blood. In order to study the rheological behaviour non-Newtonian fluids, often the routine system of calculation used, namely, the Navier Stokes equation, alone cannot be used for an in-depth study of the said behaviour or properties; more options are needed for a range of reasons; therefore, various models like Carreau, Power Law, Ellis and Cross Models are commonly used to predict the rheological properties of such fluids.

In 1929 , Williamson 1 presented a theory to study the pseudoplatic materials and formulated a constitutive equation to explain the flow characteristics of the pseudoplastic fluids, and the experiments he conducted based on these equations validated the results on the flow characteristics. Recently, these equations have garnered the attention of researchers, as the researchers have come to know that these equations can be put to use to assess/ study the blood flow problems. Many researchers have made valuable contributions in this regard.

Williamson's fluids ${ }^{2}$ were investigated using the effects of chemical reaction over steady, viscoelastic

${ }^{*}$ Author for correspondence 
boundary layer flow. Methods like killer box $\underline{\underline{3}}$ were used to obtain numerical solutions to be used in assessing the homogeneous/heterogeneous chemical reactions of boundary-layer flow of Williamson fluids flowing over a smooth stretching cylinder. Another study 4 has numerically examined the behavior of variable thermal conductivity and heat generation/absorption on the steady, incompressible boundary-layer flow of Williamson fluids.

One study ${ }^{5}$ predicted that in the case of the steadystate solutions of the momentum equation for the fluid flow derived by an infinite disk in radial direction, the centrifugal forces balanced out so the fluid flowed outward in the absence of the pressure gradient. The rotating disk in such cases is expected to be working like a centrifugal fan, and the fluid flow originating from the rotating disk is replaced by an axial one that springs back on the surface of the rotating disk.

The reflection of the non-Newtonian fluid flowing over a rotating disk presented a great challenge to researchers in the field. The non-Newtonian flow of fluid over a rotating disk is a significant problem that needed immediate resolutions because of its extensive use in various fields, including engineering, chemical, and electro-medical processes. Recent research $\frac{6-7}{}$ on the said problems pointed out a range of outcomes, including the non-Newtonian laminar flow, double diffusion effect on unsteady flow, head transfer over a rotating disk, as well as the boundary-layer stability of non-Newtonian fluid flow

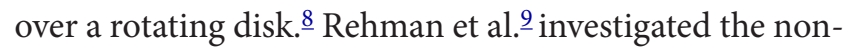
Newtonian Casson fluid flow problem over a semi-infinite disk and analyzed the characters of both thermophores and Brownian motion parameters for nanosized particles inside the magnetized Casson liquid. The problem of two turbulent simulations with overlapping small and large Reynolds numbers was reported to be a result of direct numerical simulations performed for the turbulent rotating-disk boundary layer. .0 The study by Khan et al. $\underline{11}$ investigated the problem of stress fluid flow occurring as a result of rotation of a disk.

In many industrial, chemical and power generation processes, massive amount heat is generated. In order to protect the machines operating in such high temperatures, appropriate cooling systems are to be in place to keep the machinery cool and control the heat and keep it at a level within which the machinery can perform unhindered and do not break down. Thus, different coolants like water, ethylene glycol and engine oils of different grades are used.
However, it is a proven fact that the thermal conductivity of liquids is way below that of metallic solids; due to this, all these coolants can never achieve cooling on a level that metallic solids can. In order to achieve the desired goal of reducing and maintaining temperature at an ideal level to keep the machinery functioning, a small amount of metallic nanoparticles are added to these coolants, which greatly increases the heat-dissipating capability of these coolants. The nanoparticles added to these coolants are made of Copper $(\mathrm{Cu})$, Alumina $\left(\mathrm{Al}_{2} \mathrm{O}_{3}\right)$ and titania $\left(\mathrm{TiO}_{2}\right)$, or nano tubes, as described by Tiwari and Das and Lotfi et al. $\underline{12-14}$

The aim of the present study was to analyze the features of the boundary-layer flow and heat transfer over a rotating porous disk placed in a Williamson nanofluid. The study aimed analyse the effects of three kinds of nanoparticles: Copper $(\mathrm{Cu})$, Alumina $\left(\mathrm{Al}_{2} \mathrm{O}_{3}\right)$ and titania $\left(\mathrm{TiO}_{2}\right)$. Authors have proposed different models for nano fluids, like those proposed by Buongiorno and others. $\underline{15-18}$ Oztop and Abu-Nada $\frac{19}{9}$ have analyzed the behaviour of viscous nanofluid flow of the boundary layer's laminar flow and heat transfer over a rotating porous disk. The present study was conducted using the model devised by Tiwari and Das. ${ }^{13} \mathrm{~A}$ set of nonlinear differential equations were incorporated in the homotopy analysis method (HAM) used by the study. The conduct of nanoparticles and suction/injection parameters over the fluid flow and heat transfer are presented and discussed.

\section{Mathematical Formulation}

The study analysed the effects of steady, incompressible and axially symmetric flow of Williamson fluid over a rotating porous disk immersed in a nanofluid. Figure 1 shows the schematic diagram of the problem discussed.

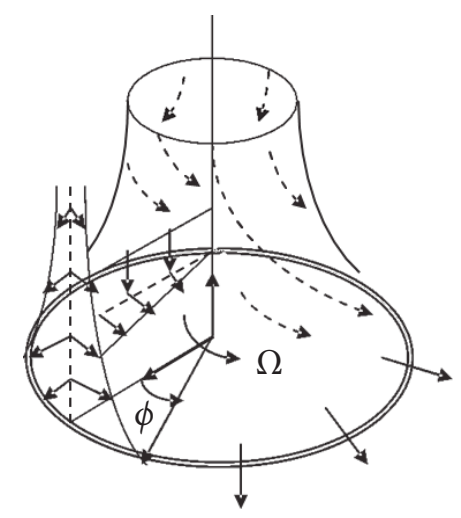

Figure 1. Physical model of the problem. $\frac{16}{6}$ 
Let the cylindrical coordinates $(r, \phi, z)$ be chosen such that the radial component $r$ is along the length of the disk. The porous disk is assumed to be rotating with a constant angular velocity $\Omega$ and extends with initial position $z=0$, in the space $z>0$ of the disk to infinity. The fluid velocity components in the increasing direction of coordinates are taken as $u, v, w$. The surface constant temperature is assumed to be $\breve{T}_{w}$. The Williamson fluid has a constant pressure $\breve{P}_{\infty}$ and a uniform ambient temperature $\bar{T}_{\infty} \cdot \bar{w}_{0}$ is taken as the constant mass flux applied at the disk surface.

Under the stated assumptions, the governing system of partial differential equations for the case of steady, incompressible laminar boundary-layer flow of Williamson nanofluid are

$$
\begin{gathered}
\frac{\partial u}{\partial r}+\frac{u}{r}+\frac{\partial w}{\partial z}=0 \\
u \frac{\partial u}{\partial r}+w \frac{\partial u}{\partial z}-\frac{v^{2}}{r}+\frac{1}{\breve{\rho}_{n f}} \frac{\partial p}{\partial r} \\
=2 \frac{\breve{\mu}_{n f}}{\breve{\rho}_{n f}}\left[\frac{\partial^{2} u}{\partial r^{2}}+\frac{1}{r}\left(\frac{\partial u}{\partial r}\right)-\frac{u}{r^{2}}+\frac{1}{2} \Gamma\left[\frac{\partial u}{\partial z} \frac{\partial^{2} u}{\partial z^{2}}+\frac{\partial w}{\partial r} \frac{\partial^{2} u}{\partial z^{2}}\right]\right] \\
u \frac{\partial v}{\partial r}+w \frac{\partial v}{\partial z}+\frac{u v}{r}=\frac{\breve{\mu}_{n f}}{\breve{\rho}_{n f}}\left[\frac{\partial^{2} v}{\partial r^{2}}+\frac{1}{r} \frac{\partial v}{\partial r}-\frac{v}{r^{2}}+\Gamma\left[\frac{\partial v}{\partial z} \frac{\partial^{2} v}{\partial z^{2}}\right]\right] \\
u \frac{\partial w}{\partial r}+w \frac{\partial w}{\partial z}+\frac{1}{\breve{\rho}_{n f}} \frac{\partial p}{\partial z}=\frac{\breve{\mu}_{n f}}{\breve{\rho}_{n f}}\left[\Gamma\left[\frac{\partial}{\partial r}\left(\frac{\partial u}{\partial z}\right)^{2}+\frac{\partial u}{\partial z} \frac{\partial^{2} w}{\partial r^{2}}\right]\right. \\
\left.+\frac{1}{r}+\frac{\Gamma}{r}\left[\left(\frac{\partial u}{\partial z}\right)^{2}+\frac{\partial u}{\partial z} \frac{\partial w}{\partial r}\right]+2 \frac{\partial^{2} w}{\partial z^{2}}\right], \\
u \frac{\partial \breve{T}}{\partial r}+w \frac{\partial \breve{T}}{\partial z}=\breve{\alpha}_{n f}\left(\frac{\partial^{2} \breve{T}}{\partial r^{2}}+\frac{1}{r} \frac{\partial \breve{T}}{\partial r}+\frac{\partial^{2} \breve{T}}{\partial z^{2}}\right)
\end{gathered}
$$

The related boundary conditions of the problem are

$$
\begin{gathered}
u=0, v=\Omega r, w=w_{0}, \breve{T}=\breve{T}_{w} \text { at } z=0, \\
u \rightarrow 0, v \rightarrow 0, \breve{T} \rightarrow \breve{T}_{\infty}, \breve{P} \rightarrow \breve{P}_{\infty} a s z \rightarrow \infty,
\end{gathered}
$$

where $\breve{T}$ and $\breve{T}_{\infty}$ denote the temperature of fluid and ambient fluid, respectively. $\breve{P}$ and $\breve{P}_{\infty}$ denote the pressure of fluid and ambient fluid, respectively. $w_{0}$ shows velocity of suction or injection, $\breve{\mu}_{n f}$ represents the dynamics viscosity, $\breve{\alpha}_{n f}$ is the thermal diffusivity and $\breve{\rho}_{n f}$ is the density of the fluid. The following assumptions are made following those reported in Patel et al. and others..$\underline{2021}$

$$
\begin{gathered}
\breve{\mu}_{n f}=\frac{\breve{\mu}_{f}}{(1-\varphi)^{\frac{5}{2}}}, \breve{\alpha}_{n f}=\frac{\breve{k}_{n f}}{\left(\breve{\rho} \breve{C}_{p}\right)_{n f}}, \breve{\rho}_{n f}=(1-\varphi) \breve{\rho}_{f}+\varphi \breve{\rho}_{s}, \\
\left(\breve{\rho} \breve{C}_{p}\right)_{n f}=(1-\varphi)\left(\breve{\rho}_{p}\right)_{f}+\varphi\left(\breve{\rho} \breve{C}_{p}\right)_{s}, \\
\frac{\breve{k}_{n f}}{\breve{k}_{f}}=\frac{\left(\breve{k}_{s}+2 \breve{k}_{f}\right)-2 \varphi\left(\breve{k}_{f}-\breve{k}_{s}\right)}{\left(\breve{k}_{s}+2 \breve{k}_{f}\right)+\varphi\left(\breve{k}_{f}-\breve{k}_{s}\right)}=1+(1+c P e) \frac{\breve{k}_{s} \breve{A}_{s}}{\breve{k}_{f} \breve{A}_{f}}
\end{gathered}
$$

Where $\left(\breve{\rho} \breve{C}_{p}\right)_{n f}$ and $\breve{k}_{n f}$ are heat capacitance and effective thermal conductivity of the nanofluid. The subscripts $f$ and $s$ are used for the fluid and the solid,c represents the experimental constant, while the Péclet numberis $P e=\frac{u_{s} d_{s}}{\breve{\alpha}_{f}}$, also $\frac{\breve{A}_{s}}{\breve{A}_{f}}=\frac{\varphi}{1-\varphi} \frac{d_{f}}{d_{s}}$, where $d_{f}$ and $d_{s}$ are, respectively, molecular sizes of the fluid and the diameter of spherical solid nanoparticles(taken as $d_{s}=38$ nm and $\left.d_{f}=2 \dot{A}\right) . \quad u_{s}=\frac{2 \breve{k}_{b} T}{\pi \breve{\mu}_{f} d_{s}^{2}} \quad$ is the Brownian motion velocity of solid particles,represented as $\breve{k}_{b}=1.3807 \times$ $10^{-23} \mathrm{~J} / \mathrm{K}$, which is Boltzmann constant.

The next equation that was applied was the Von Karman transformation.

$$
\begin{gathered}
u=r \Omega F(\eta), v=r \Omega G(\eta), w=\sqrt{\Omega v_{f}} H(\eta), \\
\eta=\sqrt{\frac{\Omega}{v_{f}}} z, \breve{p}-\breve{p}_{\infty}=-\rho_{f} v_{f} \Omega \breve{\mathrm{P}}(\eta), \theta(\eta)=\frac{\breve{T}-\breve{T}_{\infty}}{\breve{T}_{w}-\breve{T}_{\infty}},
\end{gathered}
$$

Substituting the above transformation in Eqs. (1)-(7) to convert these partial differential equations into a system of ordinary differential equations, we have

$$
\begin{aligned}
& \frac{\partial \mathrm{H}}{\partial \eta}+2 \mathrm{~F}=0, \\
& \sqrt{\operatorname{Re}} W e \frac{1}{(1-\varphi)^{\frac{5}{2}}\left(1-\varphi+\varphi \frac{\breve{\rho}_{s}}{\check{\rho}_{f}}\right)} \frac{\partial^{2} \mathrm{~F}}{\partial \eta^{2}} \frac{\partial \mathrm{F}}{\partial \eta}-\mathrm{H} \frac{\partial \mathrm{F}}{\partial \eta}-\mathrm{F}^{2}+G^{2}=0, \\
& \sqrt{\operatorname{Re}} W e \frac{1}{(1-\varphi)^{\frac{5}{2}}\left(1-\varphi+\varphi \frac{\rho_{s}}{\rho_{f}}\right)} \frac{\partial^{2} \mathrm{G}}{\partial \eta^{2}} \frac{\partial \mathrm{G}}{\partial \eta}-\mathrm{H} \frac{\partial \mathrm{G}}{\partial \eta}-2 \mathrm{FG}=0
\end{aligned}
$$




$$
\frac{1}{P_{r}} \frac{\breve{k}_{n f} / \breve{k}_{f}}{\left(\breve{\rho} \breve{C}_{p}\right)_{n f} /\left(\breve{\rho}_{p}\right)_{f}} \frac{\partial^{2} \theta}{\partial \eta^{2}}-\mathrm{H} \frac{\partial \theta}{\partial \eta}=0,
$$

with non-dimensional boundary conditions,

$$
\begin{gathered}
F(0)=0, G(0)=1, H(0)=h_{0}, \theta(0)=1, \\
F(\infty)=0, G(\infty)=0, \theta(\infty)=0,
\end{gathered}
$$

where $R e=\frac{(\Omega r) r}{v_{f}}$ is the Reynolds number associated with the fluid motion, $W e=\Omega \Gamma$ is the Weissenberg number, $\operatorname{Pr}=\frac{\breve{C}_{p} \breve{\mu}_{f}}{\breve{k}_{f}}$ is the Prandtl number and $h_{0}=\frac{w_{0}}{\sqrt{\Omega v_{f}}}$ is constant velocity of suction/injection. And $h_{0}<0$ is the velocity of suction and $h_{0}>0$ is the velocity of injection. The coefficient of skin friction in tangential and radial directions $\breve{C}_{f \phi}$ and $\breve{C}_{f r}$ and the Nusselt number are given by

$$
\breve{C}_{f \phi}=\frac{\tau_{w \phi}}{\breve{\rho}_{f}(\omega r)^{2}}, \breve{C}_{f r}=\frac{\tau_{w r}}{\breve{\rho}_{f}(\omega r)^{2}}, N u=\frac{q_{w}}{\breve{k}_{f}\left(T_{w}-T_{\infty}\right)}
$$

where

$$
\tau_{w \phi}=\breve{\mu}_{n f}\left(\frac{\partial v}{\partial z}+\frac{1}{r} \frac{\partial w}{\partial \phi}\right)_{z=0}, \tau_{w r}=\breve{\mu}_{n f}\left(\frac{\partial u}{\partial z}+\frac{1}{r} \frac{\partial w}{\partial \phi}\right)_{z=0},
$$

are transversal and radial shear stresses at the disk surface, respectively. The surface heat flux $q_{w}$ is given by

$$
q_{w}=-\breve{k}_{n f}\left(\frac{\partial \breve{T}}{\partial z}\right)_{z=0}
$$

Using Eqs.(9) and (18) in Eqs. (19) and (20) we obtain

$$
\begin{aligned}
& \sqrt{\operatorname{Re}} \breve{C}_{f r}=\frac{1}{(1-\varphi)^{\frac{5}{2}}} F^{\prime}(0), \sqrt{\operatorname{Re}} \breve{C}_{f \phi}=\frac{1}{(1-\varphi)^{\frac{5}{2}}} G^{\prime}(0), \\
& \sqrt{\operatorname{Re}} N u=-\frac{1}{r} \frac{\breve{k}_{n f}}{\breve{k}_{f}} \theta^{\prime}(0),
\end{aligned}
$$

\section{HAM Solution}

The system of nonlinear ordinary differential Eqs. (13)(16) subjected to boundary conditions in equations $\frac{17,18}{}$ has been solved by using the method of homotopy analysis $\frac{22-50}{2}$ taking into account the different values of fraction parameter $\varphi$ and velocity (suction/injection) parameter $h_{0}$. Three types of solid nanoparticles of spherical shape, namely copper $(\mathrm{Cu})$, alumina $\left.\left(\mathrm{Al}_{2} \mathrm{O}_{3}\right)\right)$ and titania $\left(\mathrm{TiO}_{2}\right)$, are considered. To achieve the boundary conditions at infinity, $\eta_{\infty}$ the boundarylayer thickness, has been studied for different values of parameters $\varphi$ and $h_{0}$ for the functions $H(\eta), F(\eta), G(\eta)$ and $\theta(\eta)$. To solve the system using the homotopy analysis we made initial estimates for $H_{0}(\eta), F_{0}(\eta), G_{0}(\eta)$ and $\theta_{0}$ $(\eta)$ as

$$
H_{0}(\eta)=0, F_{0}(\eta)=\eta e^{(-\eta)}, G_{0}(\eta)=e^{(-\eta)}, \theta_{0}(\eta)=e^{(-\eta)} .
$$

and the auxiliary operators $\mathcal{L}_{1}(H), \mathcal{L}_{2}(F), \mathcal{L}_{3}(G)$ and $\mathcal{L}_{4}(\theta)$ are

$$
\begin{gathered}
\mathcal{L}_{1}(H)=H^{\prime}=\frac{d H}{d \eta}, \mathcal{L}_{2}(F)=F^{\prime \prime}+F^{\prime}=\frac{d^{2} F}{d \eta^{2}}+\frac{d F}{d \eta}, \\
\mathcal{L}_{3}(G)=G^{\prime \prime}+G^{\prime}=\frac{d^{2} G}{d \eta^{2}}+\frac{d G}{d \eta}, \mathcal{L}_{4}(\theta)=\theta^{\prime \prime}+\theta^{\prime}=\frac{d^{2} \theta}{d \eta^{2}}+\frac{d \theta}{d \eta},
\end{gathered}
$$

This follows

$$
\begin{gathered}
\mathcal{L}_{1}\left(c_{1}\right)=0, \mathcal{L}_{2}\left(c_{2} e^{-\eta}+c_{3}\right)=0, \\
\mathcal{L}_{3}\left(c_{4} e^{-\eta}+c_{5}\right)=0, \mathcal{L}_{4}\left(c_{6} e^{-\eta}+c_{7}\right)=0,
\end{gathered}
$$

The first-order homotopy approximations are as follows:

$$
H_{1}(\eta)=2 \hbar-2 e^{(-\eta)} \hbar+h_{0}-2 \eta e^{-\eta} \hbar,
$$

$$
\begin{aligned}
F_{1}(\eta)=- & \frac{5}{4} e^{-2 \eta} \hbar+-\frac{5}{4} e^{-\eta} \hbar+e^{-\eta} \eta-\frac{3}{2} e^{-2 \eta} \hbar \eta \\
- & \frac{1}{2} e^{-2 \eta} \hbar \eta^{2}-\frac{1}{2} e^{-\eta} \hbar \eta^{2} h_{0}-\frac{1}{2} e^{-2 \eta} \hbar \sqrt{R e} W e \phi_{1}+ \\
& \frac{1}{2} e^{-\eta} \hbar \sqrt{R e} W e \phi_{1}-\frac{1}{2} e^{-2 \eta} \hbar \sqrt{R e} W e \eta^{2} \phi_{1}, \\
G_{1}(\eta)= & -\frac{3}{2} e^{-2 \eta} \hbar+\frac{3}{2} e^{-\eta} \hbar-e^{-2 \eta} \hbar \eta-e^{-\eta} \hbar h_{0} \eta \\
& +e^{-\eta}-\frac{1}{2} e^{-2 \eta} \hbar \sqrt{R e} W e \phi_{1}+\frac{1}{2} e^{-\eta} \hbar \sqrt{R e} W e \phi_{1} \\
& \theta_{1}(\eta)=e^{-\eta}-e^{-\eta} \hbar h_{0} \eta-\frac{K r}{P_{r}} e^{-\eta} \hbar \eta,
\end{aligned}
$$


where $\phi_{1}=\frac{1}{(1-\varphi)^{\frac{5}{2}}\left(1-\varphi+\varphi \frac{\breve{\rho}_{s}}{\breve{\rho}_{f}}\right)}$, and $K r=\frac{\breve{k}_{u f} / \breve{k}_{f}}{(\breve{\rho} \breve{C} p)_{u f} /(\breve{\rho} \breve{C} p)_{f}}$

The higher-order iterations are obtained through the use of symbolic software MATHEMATICA.

\section{Results and Discussion}

Table 1 contains some important thermophysical properties of Copper $(\mathrm{Cu})$, Alumina $\left(\mathrm{Al}_{2} \mathrm{O}_{3}\right)$ and Titania $\left(\mathrm{TiO}_{2}\right)$ nanoparticles used in the analysis whose findings have been repoted here. Tables $2-3$ correspond to the radial skin friction coefficient $\breve{C}_{f r}$ and tangential skin friction coefficient $\breve{C}_{f \phi}$ computed against different values of $\varphi$ and $h_{0}$. Table 4 presents values for the rate of heat transfer $-\theta^{\prime}(0)$ that corresponds to the Nusselt numbers $N u$ presented for different values of $\varphi, P r$ and $h_{0}$.

The behavior of non-dimensional velocity profiles $F(\eta)$, $H(\eta)$ and $G(\eta)$ and the non-dimensional temperature profile $\theta(\eta)$ are plotted in Figures 2-7 for different combinations of the physical parameters used in the study. Figure1 shows the patterns in the differenes identified for the three velocity profiles $F(\eta), H(\eta)$ and $G(\eta)$. Figures 3-5 illustrate the behavior of non-dimensional velocity profiles $F(\eta), H(\eta)$ and $G(\eta)$ plotted for different values of the surface suction/injection velocity $h_{0}$. The velocity profiles increased with an increase in the values of suction/injection velocity $h_{0}$. Figure 6 shows the behavior of non-dimensional temperature profile $\theta(\eta)$ for different values of the surface suction/injection velocity $h_{0}$. From the graph, it can be noted that the non-dimensional velocity $\theta(\eta)$ increases correspondiong to an increase in surface suction/injection velocity, $h_{0}$. Figure 7 Shows the behavior of non-dimensional temperature profile $\theta(\eta)$ for different values of $\varphi$. From the figure it can be observed

Table 1. Thermophysical properties of nanoparticles

\begin{tabular}{|l|l|c|l|}
\hline Physical properties & $\mathrm{Cu}$ & $\mathrm{Al}_{2} \mathrm{O}_{3}$ & $\mathrm{TiO}_{2}$ \\
\hline$\breve{C}_{p}(\mathrm{~J} / \mathrm{kgK})$ & 385 & 765 & 686.2 \\
\hline$\breve{\rho}\left(\mathrm{kg} / \mathrm{m}^{3}\right)$ & 8933 & 3970 & 4250 \\
\hline$\breve{k}(\mathrm{~W} / \mathrm{mK})$ & 400 & 40 & 8.9538 \\
\hline
\end{tabular}

that an increase in $\varphi$ produces a minute increase in the non-dimensional temperature profile $\theta(\eta)$.

Table 2. Numerical values of radial skin friction coefficient $F^{\prime}(0)$ for different values of $\varphi$ and $h_{0}$

\begin{tabular}{|l|c|l|l|l|}
\hline$\varphi$ & $h_{0}$ & \multicolumn{1}{|c|}{$\mathrm{Cu}$} & $\mathrm{Al}_{2} \mathrm{O}_{3}$ & $\mathrm{TiO}_{2}$ \\
\hline \multirow{4}{*}{0} & -2 & 1.04348 & 1.04348 & 1.04348 \\
\cline { 2 - 5 } & -1 & 1.00834 & 1.00834 & 1.00834 \\
\cline { 2 - 5 } & 0 & 0.991665 & 0.991665 & 0.991665 \\
\cline { 2 - 5 } & 1 & 0.976298 & 0.976298 & 0.976298 \\
\hline \multirow{3}{*}{0.1} & 2 & 0.863348 & 0.863348 & 0.863348 \\
\cline { 2 - 5 } & -1 & 1.01803 & 1.01803 & 1.01803 \\
\cline { 2 - 5 } & 0 & 0.927579 & 0.927579 & 0.927579 \\
\cline { 2 - 5 } & 1 & 0.979547 & 0.979547 & 0.979547 \\
\hline
\end{tabular}

Table 3. Numerical values of tangential skin friction coefficient $-G^{\prime}(0)$ for different values of $\varphi$ and $h_{0}$

\begin{tabular}{|l|c|l|l|l|}
\hline$\varphi$ & $h_{0}$ & $\mathrm{Cu}$ & $\mathrm{Al}_{2} \mathrm{O}_{3}$ & $\mathrm{TiO}_{2}$ \\
\hline \multirow{4}{*}{0} & -2 & 3.56816 & 3.56816 & 3.56816 \\
\cline { 2 - 5 } & -1 & 3.40462 & 3.40462 & 3.40462 \\
\cline { 2 - 5 } & 0 & 1.4771 & 1.4771 & 1.4771 \\
\cline { 2 - 5 } & 1 & 0.835435 & 0.835435 & 0.835435 \\
\cline { 2 - 5 } & 2 & 0.677855 & 0.677855 & 0.677855 \\
\hline \multirow{4}{*}{0.1} & -1 & 3.50746 & 3.50746 & 3.50746 \\
\cline { 2 - 5 } & 0 & 1.7581 & 1.7581 & 1.7581 \\
\cline { 2 - 5 } & 1 & 0.851297 & 0.851297 & 0.851297 \\
\hline
\end{tabular}

Table 4. Numerical values of the rate of heat transfer $-\theta^{\prime}(0)$ for different values of $\varphi, \operatorname{Pr}$ and $h_{0}$

\begin{tabular}{|l|c|c|c|c|c|}
\hline$\varphi$ & $P r$ & $h_{0}$ & $\mathrm{Cu}$ & $\mathrm{Al}_{2} \mathrm{O}_{3}$ & $\mathrm{TiO}_{2}$ \\
\hline \multirow{4}{*}{0} & \multirow{3}{*}{0.64} & -1 & 0.869333 & 0.869333 & 0.869333 \\
\cline { 3 - 6 } & \multirow{3}{*}{0.71} & 1 & 0.532922 & 0.532922 & 0.532922 \\
\cline { 3 - 6 } & & -2 & -0.407414 & -0.407414 & -0.407414 \\
\cline { 3 - 6 } & & 2 & 0.485699 & 0.485699 & 0.485699 \\
\cline { 3 - 6 } & \multirow{3}{*}{6.2} & 0 & 0.965542 & 0.965542 & 0.965542 \\
\hline \multirow{3}{*}{0.1} & \multirow{3}{*}{6.2} & 1 & -0.238215 & -0.238215 & -0.238215 \\
\hline & & -1 & 0.436482 & 0.436482 & 0.436482 \\
\hline & & 1 & 1.0439 & 1.0439 & 1.0439 \\
\hline & & 1 & 0.512261 & 0.512261 & 0.512261 \\
\hline
\end{tabular}




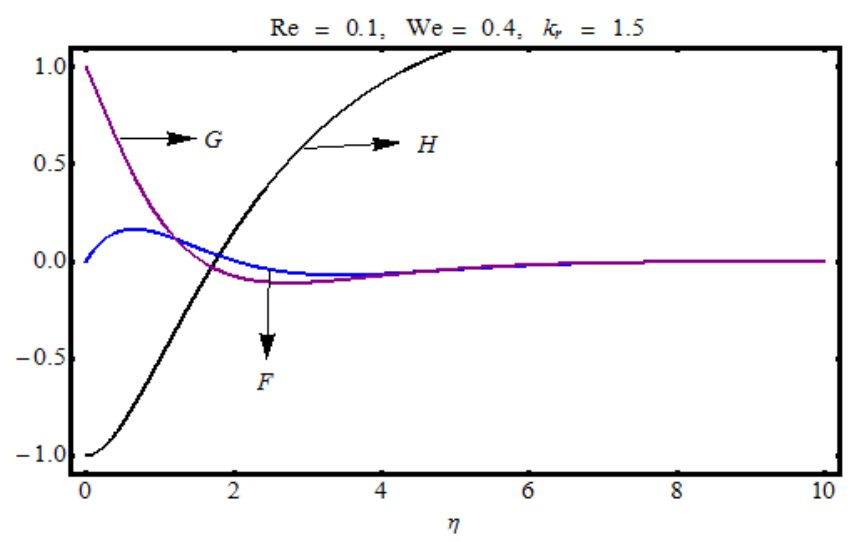

Figure 2. Profiles for $-H, F$ and $G$ for nanofluid at $\varphi=0.1$ and $h_{0}=1$.

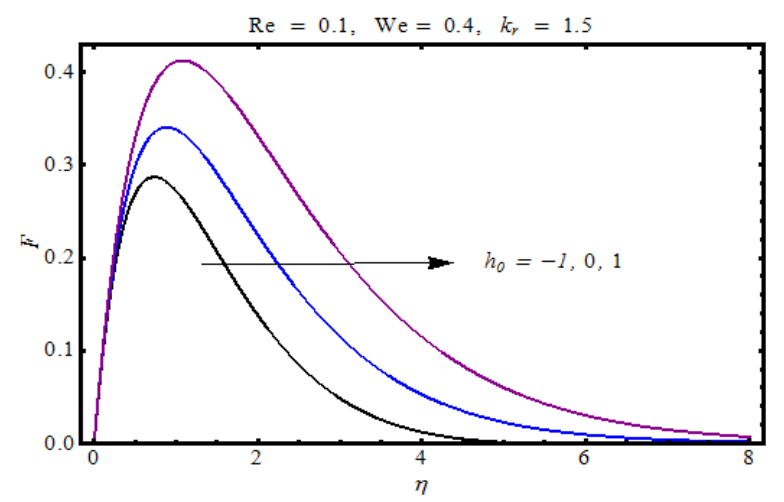

Figure 3. Profiles for radial velocity of $F(\eta)$ for different values of $h_{0}$ when $\varphi=0.1$.

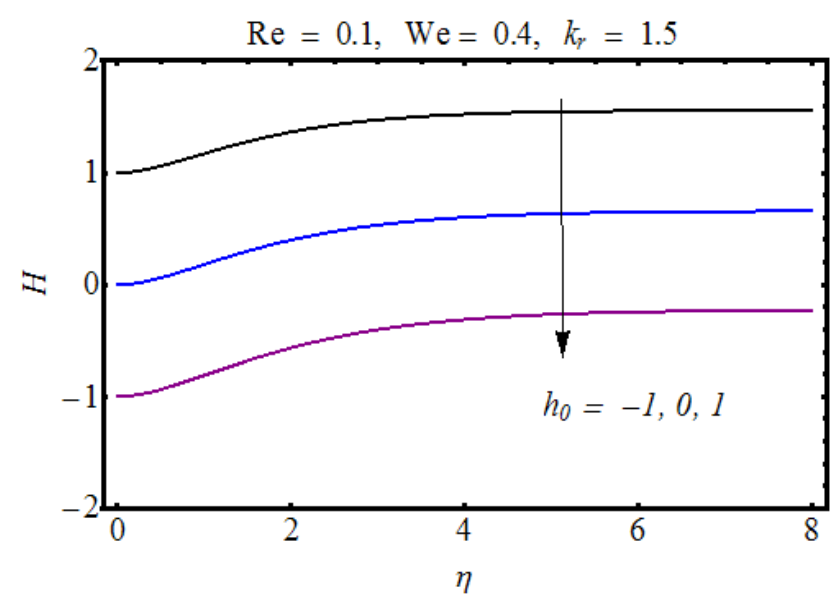

Figure 4 Profiles for axial velocity of $-H(\eta)$ for different values of $h_{0}$ when $\varphi=0.1$.

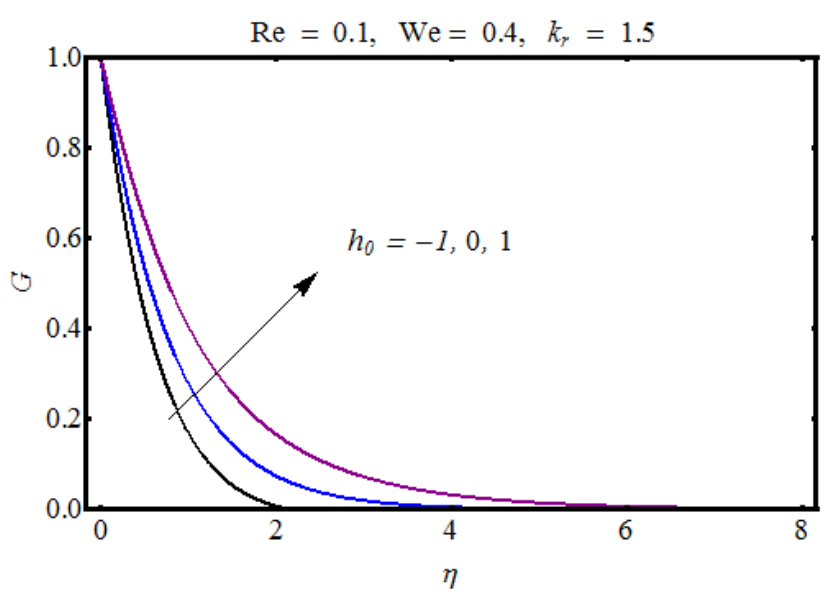

Figure 5. Profiles for tangential velocity of $G(\eta)$ for different values of $h_{0}$ when $\varphi=0.1$.

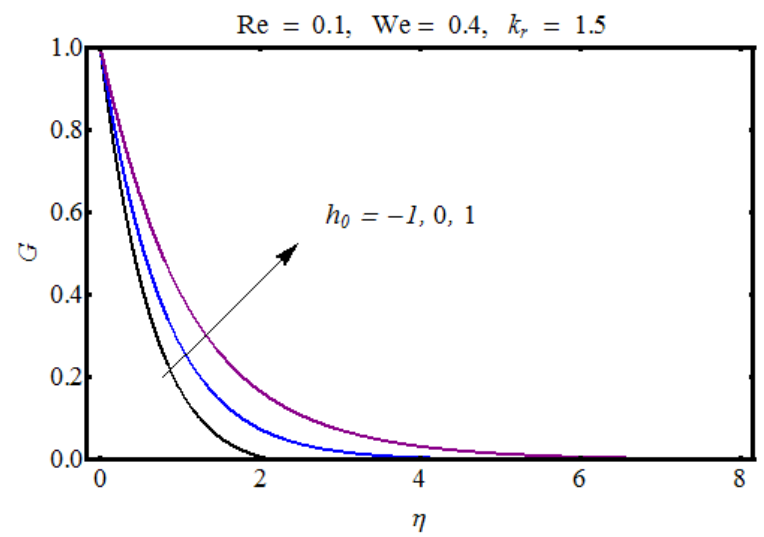

Figure 6. Profiles for temperature of $\theta(\eta)$ for different values of $h_{0}$ when $\operatorname{Pr}=6.2$ and $\varphi=0.1$.

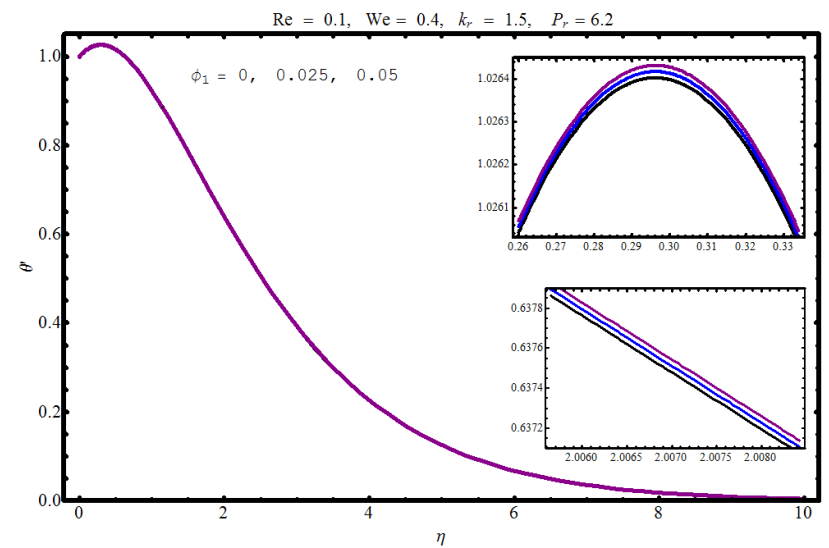

Figure 7. Profiles for temperature of $\theta(\eta)$ for different values of $\varphi_{1}$ of base fluid when $\operatorname{Pr}=6.2$ and $h_{0}=1$. 


\section{Conclusion}

The problem of a non-Newtonian boundary-layer flow and heat transfer of Williamson fluid containing nanoparticles and in motion over a rotating porous diskwas analyzed analytically using the method of homotopy analysis. The findings made and our conclusions support the use of Copper $(\mathrm{Cu})$, Alumina $\left(\mathrm{Al}_{2} \mathrm{O}_{3}\right)$ and Titania $\left(\mathrm{TiO}_{2}\right)$ nanoparticles to enhance the heat dissipation capability of coolants are presented as follows.

1. By increasing the suction/injection parameter, the non-dimension velocity profile $H(\eta)$ increases.

2. By increasing the suction/injection parameter, the non-dimension velocity profile $F(\eta)$ increases.

3. By increasing the suction/injection parameter, the non-dimension velocity profile $G(\eta)$ increases.

4. By increasing the suction/injection parameter, the non-dimension temperature function $\theta(\eta)$ increases.

\section{References}

1. Williamson RV. The flow of pseudoplastic materials, Ind Eng Chem. 1929;21:1108-11.

2. Khan NA, Khan S, Riaz F. Boundary layer flow of Williamson fluid with chemically reactive species using scaling transformation and homotopy analysis method. Math Sci Lett. 2014;3:199-205.

3. Malik MY, Salahuddin T, Hussain A, Bilal S, Awais M. Homogeneous heterogeneous reactions in Williamson fluid model over a stretching cylinder by using Keller box method. AIP Adv. 2015;5:107227.

4. Malik MY, Bibi M, Khan F, Salahuddin T. Numerical solution of Williamson fluid flow past a stretching cylinder and heat transfer with variable thermal conductivity and heat generation/absorption. AIP Adv. 2016;6:035101.

5. On laminar and turbulent friction. [cited 1946]. https:// authors.library.caltech.edu/47896/.

6. Khan NA, Aziz S, Khan NA. MHD flow of PowellEyring fluid over a rotating disk. J. Taiwan Inst Chem E. 2014;45:2859-67.

7. Khan NA, Aziz S, Khan NA. Numerical simulation for the unsteady MHD flow and heat transfer of couple stress fluid over a rotating disk. PLoS One. 2014;9:95423.

8. Griffiths PT, Stephen SO, Bassom AP, Garrett SJ. Stability of the boundary layer on a rotating disk for power-law fluids. J non-Newton Fluid Mech. 2014;207:1-6.

9. Rehman K, Malik MY, Khan WA, Khan I, Alharbi SO. Numerical solution of non-Newtonian fluid flow due to rotatory rigid disk. Symmetry. 2019;11(5): 699.
10. Appelquist E, Schlatter P, Alfredsson PH, Lingwood RJ. Turbulence in the rotating-disk boundary layer investigated through direct numerical simulations. Eur J Mech. B/Fluids. 2018;70:6-18.

11. Khan NA, Khan NA,Ullah S, Naz F. Swirling flow of couple stress fluid due to a rotating disk. Nonlinear Eng Model Appl. 2019;8:261-9.

12. Anomalously increased effective thermal conductivities of ethylene glycol-based nanofluids containing copper nanoparticles. [cited 2001]. https://aip.scitation.org/ doi/10.1063/1.1341218.

13. Tiwari RK,Das MK. Heat transfer augmentation in a twosided lid-driven differentially heated square cavity utilizing nanofluids. Int J Heat Mass Transf. 2007;50:2002.

14. Lotfi R,Saboohi Y,Rashidi AM. Numerical study of forced convective heat transfer of nanofluids: comparison of different approaches. Int Commun Heat Mass Transf. 2010;37:74.

15. Buongiorno J. Convective transport in nanofluids. J Heat Transf. 2016;128:240

16. Nield DA, Kuznetsov AV. Thermal instability in a porous medium layer saturated by a nanofluid, Int J Heat Mass Transf. 2009;52:5792.

17. Kuznetsov AV,Nield DA. Natural convective boundarylayer flow of a nanofluid past a vertical plate. Int J Therm Sci. 2010;49:243.

18. Bachok N, Ishak A, Pop I. Flow and heat transfer over a rotating porous disk in a nanofluid. Physica B. 2011;406:1767-72.

19. Oztop HF,Abu-Nada E. Numerical study of natural convection in partially heated rectangular enclosures filled with nanofluids. Int J Heat Fluid Flow. 2008;29:1326.

20. Patel HE,Sundararajan T,Pradeep T,Dasgupta A,Dasgupta N,Das SK. A micro-convectionmodel for thermal conductivity of nanofluid. Pramana J Phys. 2005;65:863.

21. The proposed homotopy analysis technique for the solution of nonlinear problems. [cited 1992]. http://www. scirp.org/(S(351jmbntvnsjt1 aadkposzje))/reference/ ReferencesPapers. aspx?ReferenceID=1569004.

22. Beyond perturbation: introduction to the homotopy analysis method. [cited 2003]. https://www.crcpress.com/ Beyond-Perturbation-Introduction-to-the-HomotopyAnalysis-Method/Liao/p/book/9781584884071.

23. Liao SJ. An explicit, totally analytic approximation of Blasius viscous flow problems. International J Non-Linear Mech. 1999;34:759-78.

24. Liao SJ. On the analytic solution of magnetohydrodynamic flows of non-Newtonian fluids over a stretching sheet. J Fluid Mech. 2003;488:189-212.

25. Liao SJ. A uniformly valid analytic solution of two dimensional viscous flow over a semi-infinite flat plate. J Fluid Mech. 1999;385:101-28. 
26. Liao SJ. On the homotopy analysis method for nonlinear problems. Appl Math Comput. 2004;147:499-513.

27. Liao SJ. Comparison between the homotopy analysis method and homotopy perturbation method. Appl Math Comput. 2005;169:1186-94.

28. Axisymmetric stagnation flow of a micropolar nanofluid in a moving cylinder. [cited 2012]. https://www.hindawi.com/ journals/mpe/2012/378259/.

29. Rehman A, Nadeem S. Mixed convection heat transfer in micropolar nanofluid over a vertical slender cylinder. Chin Phys Lett. 2012;29(12):124701-5.

30. Boundary layer flow of second grade fluid in a cylinder with heat transfer. [cited 2012]. https://www.hindawi.com/ journals/mpe/2012/640289/.

31. Nadeem S, Rehman A, Ali M. The boundary layer flow and heat transfer of a nanofluid over a vertical slender cylinder. J Nanoeng Nanosyst. 2012;226(4):165-173.

32. Nadeem S, Rehman A. Axisymmetric stagnation flow of a nanofluid in a moving cylinder. Comput Math Model. 2013;24(2):293-306.

33. Rehman A, Nadeem S, Malik MY. Stagnation flow of couple stress nanofluid over an exponentially stretching sheet through a porous medium. J Power Technol. 2013;93(2):122-32.

34. Rehman A, Nadeem S, Malik MY. Boundary layer stagnation-point flow of a third grade fluid over an exponentially stretching sheet. Braz J Chem Eng. 2013;30(3):611-8.

35. Rehman A, Nadeem S. Heat transfer analysis of the boundary layer flow over a vertical exponentially stretching cylinder. Glob J Sci Front Res. 2013;13(11):73-85.

36. Malik MY, Naseer M, Nadeem S, Rehman A. The boundary layer flow of Cassonnanofluid over a vertical exponentially stretching cylinder. Appl Nanosci. 2012;4(7):869-873.

37. Rehman A, Nadeem S, Iqbal S, Malik MY, Naseer M. Nanoparticle effect over the boundary layer flow over an exponentially stretching cylinder. J Nanoeng Nanosyst. 2014;229:17-22.

38. Malik MY, Naseer M, Nadeem S, Rehman A. The boundary layer flow of hyperbolic tangent fluid over a vertical exponentially stretching cylinder. Alex Eng J. 2014;53:747-50.

39. Malik MY, Naseer M, Nadeem S, Rehman A. Numerical study of convective heat transfer on the Power Law fluid over a vertical exponentially stretching cylinder. Appl Math Comput. 2015;4(5):346-50.

40. Rehman A, Bazai R, Achakzai S, Iqbal S, Naseer M. Boundary layer flow and heat transfer of micropolar fluid over a vertical exponentially stretched cylinder. Applied Mathematics and Computation. 2015;4(6):424-30.

41. RehmanA, Farooq G, Ahmed I, Naseer M, Zulfiqar M. Boundary layer stagnation-point flow of second grade fluid over an exponentially stretching sheet. Am J Appl Math Stat. 2015;3(6):211-9.

42. Rehmana A, Achakzai S, Nadeem S, Iqbal S. Stagnation point flow of Eyring Powell fluid in a vertical cylinder with heat transfer. J Power Technol. 2016;96(1):57-62.

43. Rehman A, Iqbal S, MohsinRaza S. Axisymmetric stagnation flow of a micropolar fluid in a moving cylinder: ananalytical solution. Fluid Mech. 2016;2(1):1-7.

44. Iftikhar N, Rehman A. Peristaltic flow of an Eyring Prandtl fluid in a diverging tube with heat and mass transfer. International J Heat Mass Transf. 2017;111:667-76.

45. Rehman A, Sheikh N. Boundary layer stagnation-point flow of micropolar fluid over anexponentially stretching sheet. Int J Fluid Mech Therm Sci. 2017;3(3):25-31.

46. Rasheed H, Rehman A, Sheikh N, Iqbal S. MHD. Boundary layer flow of nanofluid over a continuously moving stretching surface. Appl Comput Math.2017;6(6):265-70.

47. Alam Khan N, Saeed UB, Sultan F, Ullah S, Rehman A. Study of velocity and temperature distributions in boundary layer flow of fourth grade fluid over an exponential stretching sheet. AIP Adv. 2018;8:25011.

48. Iftikhar N, Rehman A, Sadaf H, Khan MN. Impact of wall properties on the peristaltic flow of $\mathrm{Cu}$-water nano fluid in a non-uniform inclined tube. Int J Heat Mass Transf. 2018;125:772-9.

49. Iftikhar N, RehmanA, Khan MN. Features of convective heat transfer on MHD peristaltic movement of Williamson fluid with the presence of Joule heating. IOP Conf Ser Mater Sci Eng. 2018;414:12010.

50. Iftikhar N, Rehman A, Sadaf $H$, Iqbal S. Study of $\left(\mathrm{Al}_{2} \mathrm{O}_{3}\right.$ \& copper)/water nanoparticles shape, slip effects and heat transfer on steady physiological delivery of MHD hybrid nanofluid. Can J Phys. 2011;97(12)1239-1252. 